\title{
Inuusinni Aqqusaaqtara - My Journey: Meeting the Information Needs of Inuit Living with Cancer
}

\author{
Savanah Ashton, Tracy Torchetti
}

\begin{abstract}
Aвstract The Inuit Cancer Project aims to help Inuit better understand cancer and improve communication between Inuit with cancer and non-Inuit healthcare providers and support services. The project has created resources that are culturally relevant, appropriate, and accessible to Inuit patients and the healthcare providers working with them. Not only are we helping to educate and empower Inuit patients and their families, but also better educating healthcare providers around the importance of cultural knowledge in cancer care. Our ultimate goal is to improve health outcomes by increasing awareness and understanding of cancer among Inuit communities. This project is a collaboration between Pauktuutit Inuit Women of Canada and the Canadian Cancer Society. It highlights how partnerships with organizations, both big and small, and connected with the community, can have a real impact.
\end{abstract}

KEYWORDS Inuit; Inuktut language; community health; cancer care; patient resources

The Inuit Cancer Project aims to help Inuit better understand cancer and improve communication between Inuit with cancer and non-Inuit doctors and support services. This five-year project is a collaboration between Pauktuutit Inuit Women of Canada (Pauktuutit) and the Canadian Cancer Society (CCS) that began in 2015.

Our partnership pairs CCS's expertise in developing accurate, evidence-based, and easy-tounderstand cancer information with Pauktuutit's expertise in community-based research and dissemination. It is an initiative that highlights how important partnerships between different organizations can collaborate to guide community-rooted projects.

As a result of this partnership, we have created a suite of resources that are accurate, culturally appropriate, and accessible to Inuit patients and the healthcare providers working with them. In phase one of the project (before CCS became a formal partner), Pauktuutit conducted focus groups with Inuit communities and experts. This research and engagement included knowledge gathering to ensure that the resources and tools were appropriately informed by Inuit culture, language and lived experience, and involved engaging and working with cultural and linguistic experts and cancer terminology content experts. This ultimately led to the production of the Kaggutiq Inuit Cancer Glossary, in English and five dialects of Inuktut. In phase two, Pauktuutit and CCS focused on the further dissemination of cancer information to Inuit. We developed a book called Inuusinni Aqqusaaqtara - My Journey, a resource for those who must travel to major centres for tests and cancer treatments. Accompanying e-learning modules are also being created to help cancer patients, their families, and service providers understand and use the content. 


\section{Why the project matters}

The cancer death rate among Inuit is high, and we want to change this.

Many factors contribute to high cancer rates among Inuit, including tobacco and alcohol use, poor diet, and physical inactivity. Furthermore, when available information is not culturally or linguistically appropriate, low cancer awareness and limited access to cancer information are also contributing factors. A lack of accessible information can prevent people from getting regular health checkups, can lead to a misunderstanding of diagnosis and treatment plans, and often means people wait to visit their doctor until they are very sick. This means culturally appropriate cancer information needs to be in a language and dialect that the patient understands (e.g. Inuktut, Inuinnaqtun, Inuvialuktun), include terms and concepts relevant to Inuit (e.g. family, country food, connection with the land, Inuit Nunangat), and contain imagery that would resonate with patients and their families. This project did just that by uniting cancer experts, Inuit, and Inuit-represented organizations to ensure the tools and resources were created by and for Inuit.

Historically, Inuit have also viewed cancer as a death sentence, largely due to poor and inaccurate translations. This, among many other reasons, has led to people not seeking treatment for treatable cancers.

A better understanding of cancer can reduce fear and empower Inuit to seek healthcare services sooner and more frequently. Often, early diagnosis and treatment means a better chance of survival. Building knowledge will help to empower not only the people with cancer but also their families and friends who want to offer support. Improved health literacy — for patients and healthcare providers - results in fewer misunderstandings and a better quality of care. By empowering Inuit with more knowledge, we hope to reduce fear and increase the likelihood that Inuit will see healthcare providers early. Ultimately, this can reduce mortality rates and improve the quality of life for Inuit living with cancer.

\section{About Pauktuutit Inuit Women of Canada}

Pauktuutit Inuit Women of Canada was incorporated in 1984 to act as the national representative organization of Inuit women in Canada. Pauktuutit addresses a range of social and health issues experienced across the 51 communities of the Canadian Arctic, along with that of Inuit in urban centres. Pauktuutit's work on health is also intended to influence policy and program development to better meet the needs of Inuit women and their families, partly by developing plain language bilingual information resources for individuals, front-line workers, and healthcare providers. We use a population health approach with a holistic view to address the social determinants of health, with language, culture, and gender as central considerations.

Pauktuutit considers the unique needs and priorities of women, men, elders, and youth in its policy and project initiatives. Our work on health issues is unique in that it seeks advice from Inuit subject matter experts and other partners through advisory committees to its projects. The resources are intended for use or modification by all Inuit communities and, whenever possible, translated into several dialects of Inuktut. Pauktuutit's health projects have addressed both the need for relevant bilingual health information and resources in the communities, 
as well as the need to build community capacity to deliver workshops and hold other events locally through train-the-trainer projects. Community health workers have told Pauktuutit that the Inuit-specific resources, and the support that they provide, give them the confidence to use the materials in their communities. We are recognized among Inuit as a credible source of health information that is more likely to be used than products produced by provincial and federal governments. The outcomes of these projects also provide strategic policy advice to the federal government on priorities and appropriate interventions.

Pauktuutit widely distributes its products to health centres and front-line health workers, land claims organizations, regional health boards, government partners, and other stakeholders. Our work is also widely promoted through conferences and other public events.

\section{About the Canadian Cancer Society}

The Canadian Cancer Society is a national, community-based organization of volunteers whose mission is to eradicate cancer and enhance the quality of life of people living with cancer. CCS is highly regarded for its cancer information resources and support programs. The goal is to provide highly credible and reliable cancer information through varied modalities

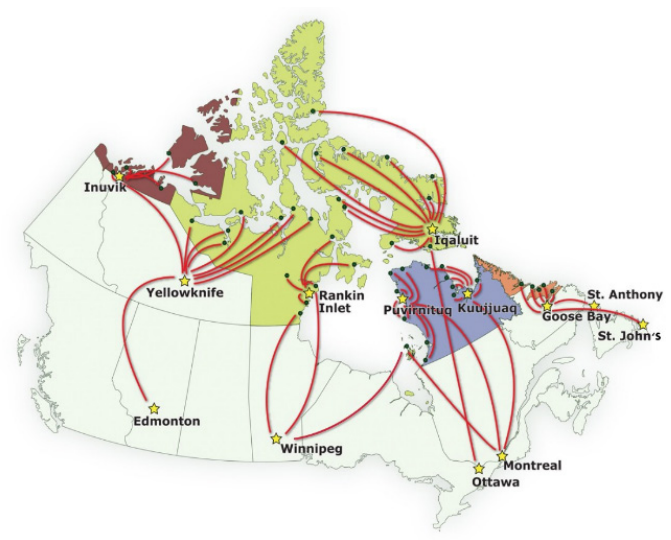

Figure 1. Map showing the great distances patients travel for care into urban centres in southern Canada and to increase the reach of programs using a person-centered and health literate approach.

A unique national infrastructure underpins the operations of information and support services. A combination of online, in-person, and telephone-based services seek to wrap around people diagnosed with cancer and their caregivers to provide barrier-free access to evidence-based and reliable information about cancer and support interventions.

CCS is committed to better understanding disparities faced by communities in accessing health information and support and to working with communities to understand how CCS products and services can be tailored or adapted to help address unmet needs. The partnership between CCS and Pauktuutit is one way to achieve this.

\section{Inuit in Canada}

Inuit are culturally, historically, and linguistically distinct from other Indigenous Peoples. There are approximately 65,000 Inuit in Canada, most of whom live in 51 communities across the North, along with a growing urban population. With a median age of only 23, Inuit are the youngest and fastest-growing population in Canada. Over the last one hundred years, Inuit have endured rapid colonization, shifting from a nomadic lifestyle to living in permanent settlements in only two generations. Various policies put in place to exercise control over Inuit included 
the removal of children from their homes and into residential schools, forced relocations to the High Arctic and other permanent settlements, and forced medical treatment, for example, removal and relocation for lengthy tuberculosis treatment. This has created intergenerational trauma for Inuit and a culture of mistrust of authority figures, including healthcare and social care providers. However, despite colonization, Inuit remain resilient and strong, and they have deep connections to land, family, language, community, and traditional knowledge, which have been sustained over thousands of years.

Today, there is a lack of human resource capacity in the communities across the spectrum of health and wellness services. Most Inuit communities are served by a health centre staffed only by nurses who rotate in and out of Arctic communities. Accessing hospitals or specialized services can require travelling thousands of miles by air from home to larger centres such as Iqaluit, Winnipeg, Edmonton, Ottawa, Montreal, or St John's (see Figure 1). In some cases, including cancer treatment, there are no community-based services equivalent to those that can be found in larger places across Canada, and specialist services are severely lacking.

Many Inuit do not have family doctors. This is due, in part, to the rotation of healthcare staff through communities. As a result, Inuit may not be able to build trusting relationships with their healthcare providers and must often repeat their medical history whenever they meet a new healthcare or service provider. This lack of access to health services also correlates to a lack of access to early testing and diagnosis. Far too often, Inuit are diagnosed at late stages of disease, when it is too late for potentially life-saving treatments.

\section{Inuit and Cancer}

The Inuktut term for cancer lacks precision. It is broadly understood as a disease that lacks a cure. This, in combination with limited health literacy about cancer and a lack of culturally appropriate cancer awareness resources, reinforces fear, stigma, and a fatalistic attitude about the disease, which in turn may discourage early screening.

Health service systems, cancer screening, and diagnostic programs and services vary between the four regions of Inuit Nunangat (Inuvialuit Settlement Region, Nunavut, Nunavik, and Nunatsiavut). Generally, diagnostic services and cancer treatment facilities are limited or non-existent in the communities, and there are no cancer clinics in Inuit Nunangat. Cultural barriers - language, lack of cultural sensitivity within the healthcare system, and a lack of culturally appropriate information resources — limit the ability of healthcare providers to meet the information needs of Inuit.

Compared to the general Canadian population, Inuit have elevated rates of nasopharyngeal, lung and bronchus, colorectal, stomach, kidney, and renal pelvis cancers. Contributing factors for high cancer incidence among Inuit include (Carrière et al., 2012):

- rapid change in lifestyle due to colonization

- socio-economic determinants of health such as low income, inadequate housing and education, food insecurity, and less access to healthcare services, including early detection screening 
- high rates of tobacco use and other modifiable risk factors such as poor diet, high alcohol use, and physical inactivity; and

- lack of access to healthcare services in remote communities, including situations where Inuit are discouraged from seeking non-urgent medical care

Also, reduced engagement in cancer screening in Inuit regions may result in cancer rates being underestimated in these regions. Potential factors in reduced access to screening include remoteness, unavailability of specialized services and screening programs, and language barriers (Inuit Tapiriit Kanatami, 2008; Tait, 2008). Furthermore, there may be an unwillingness to seek screening because the diagnosis and treatment of many cancers often requires travel outside of Inuit regions.

\section{Inuit Cancer Project, Phase 1: Focus Groups, Language Forum, Glossary}

Pauktuutit created the Inuit Cancer Project to address these problems. With financial support from the Public Health Agency of Canada, Pauktuutit partnered with the Canadian Cancer Society, the Canadian Breast Cancer Network, the Government of Nunavut and the Canadian Partnership Against Cancer to achieve the following objectives: increase Inuit knowledge (health literacy) about different cancers, cancer screening and early detection, and cancer care and treatment; increase the capacity of frontline healthcare providers to explain and promote the importance of participation in cancer screening and early detection programs; increase the understanding among non-Inuit healthcare providers about their Inuit cancer patients, Inuit culture, and Inuit attitudes about cancer and cancer treatment; and increase the cancer language/terminology capacity of all front-line healthcare providers working with Inuit.

The Inuit Cancer Project was initially a two-year initiative to develop culturally appropriate cancer awareness tools that promoted increased screening and early diagnosis of cancer among Inuit living in the Inuvialuit Settlement Region, Nunavut, Nunavik, and Nunatsiavut. The project aimed to increase awareness about cancer among Inuit to reduce the stigma associated with cancer screening and early detection and reduce the health disparities among Inuit diagnosed with the disease.

The project's early key activities included an environmental scan (literature review, online searches, and key informant interviews); nine Inuit-specific knowledge-attitude-behaviour focus group sessions; a terminology forum to develop an Inuktut glossary of cancer-related terms; and the development, translation and field testing of culturally and linguistically appropriate plain-language cancer awareness tools and complementary support material for use by Community Health Representatives/Workers and non-Inuit healthcare providers. To this end, an advisory committee with cultural, regional, administrative and subject matter expertise was established to guide the cultural content, the project's methodology and development of the final products. 


\section{Focus Groups}

The focus groups helped to develop an understanding of Inuit knowledge, attitudes, and behaviours about cancer, and they offered an opportunity to gain unique regional perspectives on how Inuit view the topic. This was an important starting point, as information campaigns are filtered through cultural lenses, and therefore, awareness products should be developed accordingly. In this case, the information gathered supported the development of educational resources and tools that better target Inuit and helped identify the best formats and media to reach Inuit and raise awareness. Furthermore, the focus groups provided an opportunity to gauge Inuit understanding of cancer in terms of what they know and feel about cancer and what behaviours and attitudes guide their reasons to participate in screening initiatives. The method allowed interaction and learning between participants and offered the potential to gather information on group consensus or diversity. Focus groups support the oral traditions of Inuit, such as face-to-face communication and sharing with community members, all of which Inuit highly value.

The focus groups confirmed that there is limited awareness among Inuit about cancer and resources. Many people do not know enough about screening and diagnosis. The lack of suitable cancer information was commonly cited as a problem. Although information is readily available in southern secondary and tertiary facilities, it is typically in English and not brought back home by Inuit cancer patients. It was found that even northern community health providers are largely unaware of the patient support services provided by treatment facilities and other organizations. For example, CCS offers a free, confidential cancer information helpline in over 100 languages, including Inuktut, and offers peer support services that can connect Inuit cancer patients with others who might have similar experiences.

\section{All my kids, husband - they were affected when I had lung cancer. The word cancer scares everyone. It's a scary topic. Even when we hear it's only a possibility.}

All the focus group sessions included individuals who had had cancer or had a family history of cancer. Participants in Nunavut and Nunavik were most likely to offer statements that cancer is incurable. The late diagnosis of cancer among Inuit probably reinforced the perception of cancer as an incurable or fatal disease. Having experienced specific cancers personally, having a family history with certain cancers, or knowing someone who had suffered from cancer seemed to be a factor in what people knew about the disease. Even so, cancer was often referred to in general terms, with no reference to a specific type.

\section{We know it can't be cured. We understand that. It scares us. It's a scary topic. We see so many people - Inuit — die from cancer so it scares me.}

In most focus group sessions, at least one participant would refer to cancer as an incurable disease. Besides fear, participants equated a cancer diagnosis with death, some expressed that they would be devastated, would give up hope, and would accept their fate. 
My mother is going to be 80 years old, she's 79 years old, yes, she's quite old. For me, I am 50 years old and I am capable of using the computer and I read and understand and can ask in English, so I would understand more about this than her.

When asked how best to inform Inuit about cancer prevention, screening and treatment, the focus groups offered a range of suggestions. Participants agreed there was limited information available to them about cancer. Some noted there was plenty of information at the cancer clinics in the south, but far less in the North. Some, however, expressed concerns about information for those who do not speak English and those who do not read. Though pamphlets were recommended, it was noted that if too long, they will not be read.

No, there will never be enough information about cancer but especially in the North. Now that you mention, I can't even think of any information provided to the public about cancer.

Participants specifically identified the need for information about different types of cancers, cancer risks, testing, treatment and diagnosis information in Inuktut for use by interpreters.

\section{Language Forum}

Before awareness tools were developed, translated, and distributed, a terminology forum was conducted in 2013 with the participation of content and language experts. It made certain that technical and cancer-related terms had suitable Inuktut-equivalent wording and contributed to the effective knowledge exchange of cancer information in each of the four Inuit regions.

There should be a different name. When we hear the word cancer, we automatically think of the worst; that it's incurable.

For me, when I hear the word cancer, I automatically assume it's something that's going to kill you. Even if it's not a deadly cancer, I still think it's going to kill you. I know it's a disease in the body but I also know often times, it's a deadly one.

There was widespread recognition that the current Inuktut term for cancer (annia aaqqijuajunnangituq, which means "incurable ailment") needed to be changed. The term invoked fear and discouraged people from seeking treatment and fighting the illness. As a result, some participants viewed cancer as an incurable disease. Others, however, recognized that cancer could be treated and managed, and they commented about the inaccuracies of the Inuktut meaning for the word "cancer." Participants in most sessions spoke about the lack of Inuktut terminology for different cancers. It was also suggested that those who cannot read or understand English need to learn about cancer verbally using Inuktut.

\section{Glossary}

The final deliverable of phase one was the Kaggutiq Inuit Cancer Glossary, a resource with cancer terminology and definitions for Inuit (see Figure 2). It is intended to provide Inuit with cancer 
and their caregivers, as well as healthcare providers, plain-language information about cancer in English, along with five dialects of Inuktut.

The cancer glossary inspired Pauktuutit and Canadian Cancer Society to find a way to continue the work of the Inuit Cancer Project. We recognized that a lack of commonly understood and used Inuktut vocabulary was a barrier to

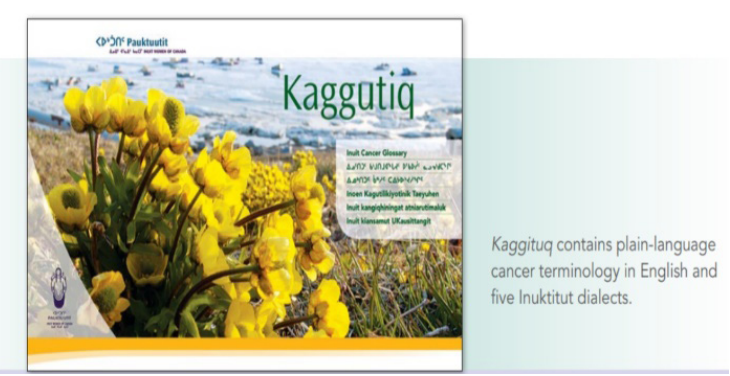

Figure 2. Inuktut-language cancer glossary communicating effectively about cancer. We also knew that working together as partners would be an effective way to combine our experience and expertise. Together, we found funding to begin phase two of the project that included the dissemination of the glossary and the development of new tools and resources to support patients, cancer survivors, family members, and healthcare providers.

\section{Inuit Cancer Project, Phase 2: Dissemination, My Journey, e-Learning}

Pauktuutit, CCS, and the project advisory committee are currently collaborating on the second phase of the Inuit Cancer Project. During the first four years, Jaguar Land Rover

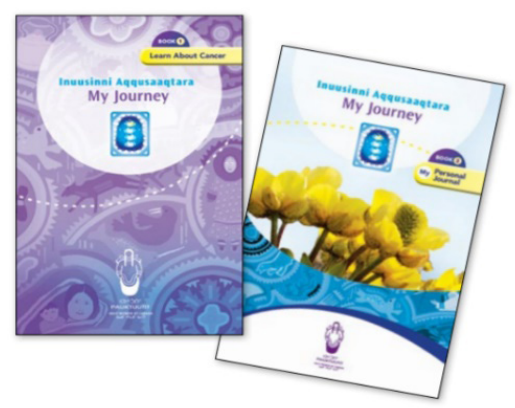

Figure 3. My Journey patient resource Canada provided funding, with the fifth and final year of the project funded by CCS. This phase focusses on developing and providing accurate information and culturally appropriate resources to support recently diagnosed Inuit through their cancer journey and improving communication among Inuit, their medical interpreters, and healthcare providers. The patient resource Inuusinni Aqqusaaqtara - My Journey has been successfully launched throughout the regions of Inuit Nunangat, with enhanced outreach and dissemination to the cancer healthcare community serving Inuit remaining a steady focus (see Figure 3).

Many Inuit with cancer prefer seeing and hearing from someone else with a similar experience rather than just relying on written information. Through consultation with our stakeholders and advisory committee, we found that while individuals found the My Journey printed resources valuable, without someone explaining its significance or how to use it, it was likely to get lost in the sea of resources given to patients during their cancer journey. To fill this gap, the project team is creating e-learning modules to help promote the My Journey resources and encourage their use of an inviting and engaging way.

E-learning will allow people to learn in different ways and at their own pace. The modules will be designed to facilitate online and offline learning to accommodate both accessibility 
within northern communities and education before participants leave their communities. The modules will also be created using the best online learning design practices to ensure learners are fully engaged and will complement the existing My Journey resources.

One e-learning module will be designed for Inuit who have recently had a cancer diagnosis and their family members/caregivers. The goal of this module is to help them feel as comfortable as possible along the journey and to encourage them to use the resources Pauktuutit and CCS have developed. It will cover the following topics:

- Learning about cancer

- How to use cancer resources

- What to expect through the cancer journey

- What to expect when going south for treatment

- Suggestions for mental wellness supports

- Examples of survivorship resources

- Sample questions to keep in mind; and

- Cultural and strength-based encouragement throughout their journey

The other e-learning module will be geared toward healthcare providers in the communities where patients live and in cities where they go for treatment. While one of our main priorities is ensuring Inuit patients and families are informed and have access to the important knowledge they need about cancer, it is important that they alone do not hold the responsibility of educating care providers regarding their histories, culture, and unique needs. This module will encourage healthcare providers to learn more about the unique needs of Inuit, cultural competency, the historical context of colonization, unresolved trauma and healing-centered engagement, and its impacts today, Inuit ways of knowing and knowledge, how to use and share the My Journey cancer resources, language, spirituality and other relevant topics.

The main goal of this module will be for healthcare providers to be able to provide cancer patients and their families with a healthcare environment where they feel respected and safe and are provided the information needed to feel as comfortable as possible throughout the treatment and recovery process. At the same time, healthcare providers will learn more about cultural competence, and the ability to demonstrate respect and empathy towards patients with diverse communication styles, different worldviews, values, and life experiences (Inuit Tuttarvingat, 2010).

\section{Conclusion}

Over the last century, Inuit have endured rapid colonization, shifting from a nomadic lifestyle to living in permanent settlements in only two generations. Various policies put in place to exercise control over Inuit include the removal of children from their homes and into residential schools, forced relocations to the High Arctic and within permanent settlements, forced medical treatment, and removal and relocation for lengthy tuberculosis treatment. This has resulted in intergenerational trauma and created a culture of mistrust by the Inuit of authority 
figures, including health and social care providers. Traditional Inuit ways of living sustained strong and healthy cultures and identities for thousands of years before colonization. Inuit remain resilient and strong and have deep connections to land, family, language, community, and traditional knowledge.

The purpose of these resources is two-fold: one, to provide information to Inuit recently diagnosed with cancer to better support them throughout their journey, and two, facilitate healthcare providers' knowledge of culturally appropriate engagement. A better understanding of cancer can reduce fear and empower Inuit to seek healthcare services sooner and more frequently. The early diagnosis and treatment are important because it often means a better chance of survival. Building knowledge will help to empower not only patients, but also their families and friends who want to offer support. If you are given resources that were made by others with similar experiences, you are more likely to use them. Improved health literacy for patients and healthcare providers — results in fewer misunderstandings and better quality of care.

In building this suite of resources, we acknowledge the importance of meaningfully engaging with the communities in which our resources are meant to serve. Meaningful engagement has many different definitions and it is up to organizations like Pauktuutit Inuit Women of Canada and the Canadian Cancer Society to ask communities not only about the best ways to do so, but also whether they feel this goal has been reached. Organizations should not see meaningful engagement as a "checkbox" or make assumptions regarding the fulfillment of that engagement; rather, engagement requires consistent conversations over long periods, like this project will continue to do. It includes face-to-face conversations and meeting with people from the community, both patients and healthcare, and service providers. It also means sustaining those relationships to build trust, just like we are trying to establish between patients and their healthcare providers.

As a result of this project, we hope Inuit will be more knowledgeable and less fearful about cancer, so they will be more likely to see their healthcare providers. We hope that, ultimately, it will contribute to reducing cancer incidence and mortality rates and improve the quality of life of Inuit living with cancer.

\section{For more information about the partnership and resources, please go to the Pauktuutit: Inuit Women of Canada website.}




\section{About the Authors}

Savanah Ashton, is the manager of health policy and programs at Pauktuutit Inuit Women of Canada. She is primarily responsible for the overall management of the health department and projects and policy initiatives related to Inuit health and well-being in arctic communities and urban hubs. With over ten years of combined experience in project management and stakeholder engagement, her priority areas include sexual and reproductive health, cancer care, and substance use and addiction.

Tracy Torchetti (corresponding author) is director of cancer information and policy at the Canadian Cancer Society. She oversees a knowledge translation and dissemination team responsible for developing cancer information on topics ranging from risk reduction to treatment, supportive care, and statistics. Her background is in medical anthropology and adult literacy. Most recently, she helped develop cancer resources for Inuit patients and healthcare professionals in Canada's Arctic. Email: Tracy.Torchetti@cancer.ca

\section{References}

Carrière, G. M., Tjepkema, M., Pennock, J., \& Goedhuis, N. (2012). Cancer patterns in Inuit Nunangat: 1998-2007. International Journal of Circumpolar Health, 71(1).

Inuit Tapiriit Kanatami. (2008). Inuit and cancer discussion paper. Ottawa, ON. Retrieved from https://www.itk.ca/wp-content/uploads/2016/07/Final-Inuit-and-Cancer-DiscussionPaper-October-2008.pdf

Inuit Tuttarvingat. (2010). Working with Inuit: Selected resources to help you learn about Inuit culture and way of life. Ottawa, ON: National Aboriginal Health Organization.

Tait, H. (2008). Aboriginal peoples survey, 2006: Inuit health and social conditions. Catalogue no. 89637-X-No. 001. Ottawa, ON: Statistics Canada. 